\title{
A multi-hop broadcast protocol design for emergency warning notification in highway VANETS
}

\author{
Muhammad Awais Javed*, Duy Trong Ngo and Jamil Yusuf Khan
}

\begin{abstract}
Multi-hop broadcast transmission is used in vehicular ad hoc networks (VANETs) to alert all vehicles within a geographical area of an emergency situation. However, the successful dissemination of multi-hop warning messages beyond the transmission range of a vehicle faces three major issues: (i) the broadcast storm, (ii) the severe interference with the existing periodic single-hop safety messages, and (iii) the hidden nodes. In this paper, we propose an efficient time-slotted multi-hop broadcast protocol that significantly reduces the number of required transmissions, while ensuring a timely and successful delivery of the warning messages. To alleviate the broadcast storm problem, we select only a subset of vehicles on the road to serve as the potential relay nodes. Each of these 'segment leaders' is responsible for forwarding the warning messages arrived in its own road segment. To avoid interfering with the safety messages transmitted periodically, we propose to allocate separate time slots for the warning messages. We also devise a signaling mechanism that ensures the reliable delivery of these multi-hop messages. Simulation results confirm that the developed protocol substantially outperforms existing schemes in terms of the number of required multi-hop transmissions and the dissemination delay. At the same time, the proposed solution maintains a high reception rate and low end-to-end delay for the single-hop safety messages.
\end{abstract}

Keywords: Emergency warning notification; Multi-hop broadcast; Signal interference; Vehicular ad hoc network

\section{Introduction}

Vehicular ad hoc network (VANET) is envisaged by the automotive industry as one of the key future technology to enhance the safety and comfort of road traffic users. VANETs could support a large number of applications including safety, traffic management, and infotainment [1-3]. The reliable working of these applications depend on efficient transmission of various type of messages with required quality of service [4-6]. In particular, safety applications are enabled by the the transmission of two main types of messages [5]. Periodic single-hop safety messages are used to support cooperative awareness applications enabling vehicles to maintain safe driving environment [7-9], whereas warning messages propagate event-driven notifications to all vehicles within a certain geographical area [10]. As the warning messages have to be transmitted

*Correspondence: muhammadawais.javed@uon.edu.au School of Electrical Engineering and Computer Science, The University of Newcastle, 2308 Callaghan, Australia to locations potentially out of the transmission range of any individual vehicle, multi-hop transmissions are necessary to support this class of traffic. Multi-hop broadcast transmission techniques have thus been developed for many vehicular emergency applications, e.g., postcrash warning, road condition warning, notification of approaching emergency vehicles, and vehicle diagnosis [10].

In multi-hop communications, broadcast storm is a serious problem. The circulation of broadcast messages around the network may consume all available bandwidth and saturate the network [11-14]. Moreover, the safety messages and the multi-hop warning messages are both transmitted on the control channel of the allocated spectrum for vehicular communications [15]. This may lead to severe interference between the two types of messages. The situation is further exacerbated by the strict packet transmission requirements of the safety messages (with a packet generation frequency of $1-10 \mathrm{~Hz}$ ) that results in hidden node collisions $[16,17]$. Since broadcast

\section{照 Springer}

(c) 2014 Awais Javed et al: licensee Springer. This is an Open Access article distributed under the terms of the Creative Commons Attribution License (http://creativecommons.org/licenses/by/4.0), which permits unrestricted use, distribution, and reproduction in any medium, provided the original work is properly credited. 
mechanism in the IEEE 802.11 standard [18] does not have a request to send (RTS) and clear to send (CTS), hidden node collisions cannot be detected. In this case, many redundant multi-hop retransmissions are required, which contribute to increasing the multi-hop dissemination delay and adversely affecting the reception rate of safety messages [19].

In this paper, we propose an efficient time-slotted multihop transmission protocol that broadcasts the emergency warning messages in a highway scenario with fewer number of transmissions generating minimum interference for the safety messages. The main contributions of the paper are as follows:

- We propose a segment leader selection mechanism to reduce the number of relay nodes. Specifically, we divide the highway into road segments of a fixed size and designate a 'segment leader' in each segment. These leaders forward the warning messages that arrive in that segment to further nodes in the propagation direction. By allowing only one potential message forwarder per road segment, the broadcast storm problem can essentially be alleviated.

- To deal with the interference from the periodic safety messages and the hidden-node problem, we propose to allocate separate multi-hop time slots to the warning messages. Here, a warning-message sender first suspends the ongoing single-hop transmissions by transmitting a black burst energy signal. After that, the warning message sender reserves the multi-hop time slot by transmitting a long-range CLEAR packet to inform all potential hidden nodes of an incoming transmission. The warning message can then be sent without being interfered by the single-hop safety messages. If there is no warning message, we allow the time slot to be used by the safety messages, thus fully utilizing the network resources.

- To further increase the reliability of the multi-hop transmission, we include a short ACK packet as part of the warning-message time slot. We also propose a mechanism to reduce the unnecessary retransmissions in the case of the ACK packet being lost.

Compared with the existing robust multi-hop protocols, namely, Distributed Vehicular Broadcast (DV-CAST) [12] and Smart Broadcast (SB) [20], our multi-hop broadcast protocol design offers a significant reduction in the number of warning message transmissions and the associated dissemination time. Furthermore, our proposed solution delivers a high reception rate and a low end-to-end delay to the periodic safety messages.

The rest of the paper is structured as follows. Section 2 reviews the current literature related to the multi-hop broadcast communications. Section 3 explains the design methodology of the proposed time-slotted multi-hop protocol. The performance analysis of the proposed multi-hop protocol is presented in Section 4. Finally, the conclusions are drawn in Section 5.

\section{Related works}

To efficiently disseminate multi-hop warning messages in VANETs, proposals in the literature can be divided into two broad categories: one that relies on the distance-based contention mechanism to select the relay node and the second category that reserves the next relay node using control packets. While the techniques that use distance based contention mechanism are simple to implement and do not require additional signaling, they incur redundant transmissions of the broadcast message especially in the presence of periodic safety messages. On the other hand, protocols using control packets reduce the number of relay nodes at the expense of additional signaling overhead. Both categories of multi-hop protocols suffer from the interference introduced by the safety messages transmitted on the same channel.

In the first category, the proposed techniques use a contention mechanism to disseminate warning messages based on the distance between a receiver and a source node. An example of this mechanism is the timer- and probability-based protocols which aim to suppress the broadcast storm [11]. Based on its respective distance to the sender of a warning message, each vehicle is assigned with a wait time or a probability of transmission. Being given the shortest wait time or the highest probability of transmission, the vehicle located furthest in the range of the sender rebroadcasts the warning message. Upon receiving this duplicate message, all other vehicles withdraw their intention to rebroadcast. The timer and probability based protocols suffer from packet collisions due to nodes selecting the same probability or wait time for transmission. This can happen when the potential relay nodes are close to each other and at a similar distance from the warning message sender.

Taking a distance-based contention approach [13] devises the contention-based forwarding protocol. Here, vehicles within a selected contention area employs a timer-based technique to disseminate the multi-hop messages. However, one weakness of this scheme is that the transmission time of potential relay nodes could get synchronized due to channel congestion, causing redundant transmissions. In [12], the proposed DV-CAST scheme employs the slotted 1-persistence approach to suppress broadcast storm and also adopts the store-carry forward mechanism for disconnected networks. The DV-CAST uses connectivity of vehicles on a road to determine if the neighborhood is well connected, sparsely connected, or totally disconnected. It then specifies routing rules 
to disseminate multi-hop message in each of the traffic density scenarios. Since DV-CAST uses timer-based technique, it has the same weakness of synchronized packet transmissions.

The proposal in [21] suggests an optimized slotted 1persistence scheme for multi-hop communications. As well, the protocol proposes the formation of vehicle clusters that includes vehicles within the transmission range of each other. Using the elected cluster front and tail vehicles, the protocol proposes a store-carry forward mechanism in sparse networks to reduce the network overhead. Synchronized transmissions and additional overhead required for cluster formation and maintenance are the drawbacks of this scheme. To consider both channel quality and transmission distance in selecting the next relay node, Slavik and Maghoub [22] assign a shorter waiting time to vehicles belonging to a connected dominating set (CDS) and employs acknowledgements for every multi-hop message. The main disadvantage of the scheme is the piggy-backed acknowledgement that is sent as part of the periodic safety message, increasing its transmission overhead. The work of [14] develops an opportunistic broadcast scheme, in which the relay nodes use a long-range ACK packet to lower the redundant multi-hop transmissions. Moreover, the dissemination of multi-hop messages takes place in two phases: first one for quick propagation of message and second one for increasing the reception reliability. However, interference from the periodic safety messages could result in loss of long-range ACK packets resulting in greater number of retransmissions.

Protocols proposed in the second category use a relay reservation mechanism with the help of control packets to suppress the broadcast storm. The urban multi-hop broadcast (UMB) protocol [23] partitions a road into small segments and lets the vehicles in the furthest nonempty segment forward the received multi-hop message. To select the furthest node and address the hidden-node problem, the source node first sends a request to broadcast (RTB) message. All nodes receiving the RTB then transmit a black burst message of duration proportional to the distance of their segment from the source. The nodes that sense the channel as busy after their black burst is over cancel their intention of rebroadcast and only the node in the furthest segment transmits a clear to broadcast message (CTB) letting the source node know of its selection as a relay node. One disadvantage of this scheme is that the vehicle selected as the relay node has to wait for the longest time during the contention phase, causing an increase in the warning notification time.

In [20], the SB protocol is devised which assigns a distance-based contention window to each road segment. A source node first sends a RTB message containing information about the contention window, segment size, and the message direction. The relay vehicle is selected as the one whose contention window expires first and it sends a CTB message that informs other vehicles to cancel their rebroadcast. On hearing the CTB message, the source node then sends the warning message to be forwarded by the selected relay node. A possible weakness of this protocol is the packet collisions caused by the selection of same backoff value for multi-hop transmission. Another proposal in [10] uses a binary partition mechanism to iteratively divide the transmission range into small segments and select the furthest relay vehicle. However, the iterative partition mechanism results in an increase of the warning notification time. For other interesting work related to routing and broadcast communication, readers are referred to [24-34].

\section{Proposed time-slotted multi-hop broadcast protocol for warning message dissemination}

The design methodology of the proposed time-slotted multi-hop broadcast protocol is explained in this section. Compared to the previous schemes discussed in Section 2, the proposed protocol introduces several new key features. First, it uses a segment leader-based message forwarding approach to reduce the number of relay vehicles. In addition, separate multi-hop time slots are allocated for the warning messages to overcome the interference with the single-hop safety messages. Also, the proposed mechanism handles the scenario of lost acknowledgement (ACK), thus effectively reducing the unnecessary retransmissions of the warning messages.

\subsection{Highway emergency warning transmission scenario}

Figure 1 illustrates a typical example of the highway VANET deployment scenario under consideration. In both directions of the road, moving vehicles are connected via wireless links to form a VANET. Assume that each vehicle is equipped with a differential global positioning system (DGPS) that can accurately measure its own position on the road. Generated by a vehicle marked as the 'emergency warning vehicle', a warning message is to be sent to other vehicles in the indicated propagation direction. As shown in Figure 1, multiple distinct warning messages could be transmitted in a VANET. Since the transmission range of a vehicle is limited, we employ a multi-hop packet transmission technique to ensure that the warning message will reach the vehicles located at long distances from the sender, i.e., the 'emergency warning vehicle'.

\subsection{Proposed approach for road segment division and segment leader selection}

To facilitate an efficient broadcast of the emergency message, we divide the highway into fixed-size road segments of length $D_{c}$ and designate one vehicle within a segment 


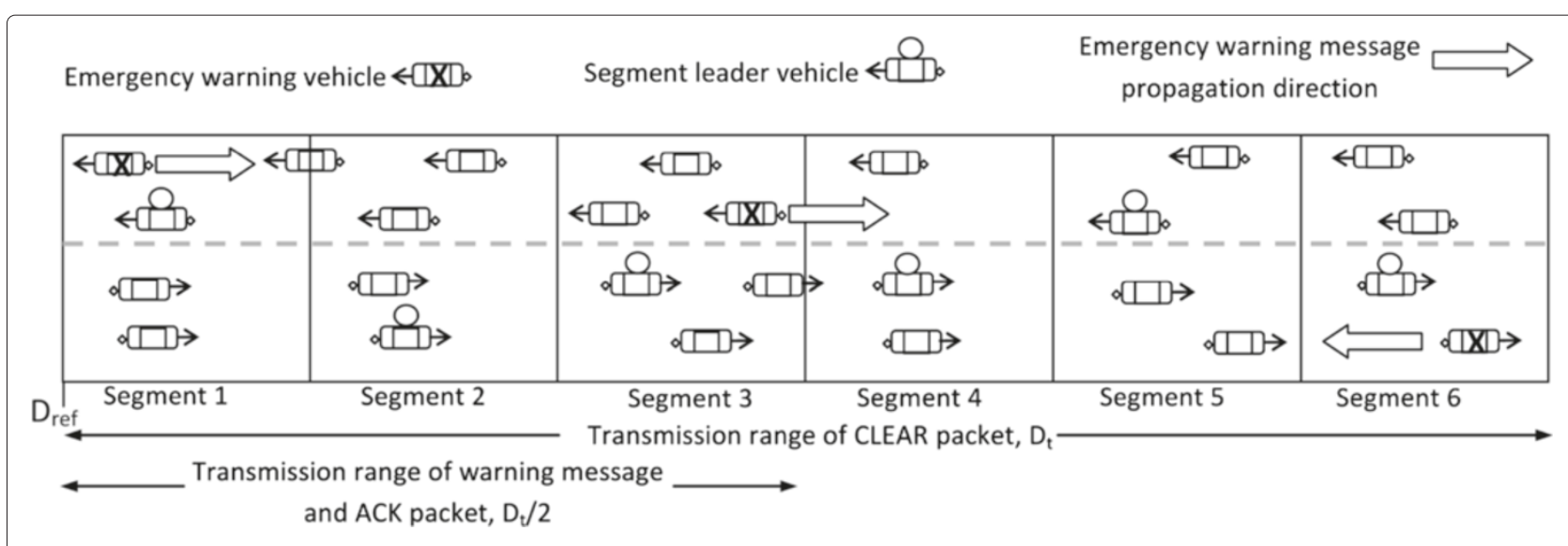

Figure 1 Warning message dissemination on a highway via a VANET.

as the 'segment leader'. This is illustrated in Figure 1. We assume that each vehicle knows its own position $D_{v}$ with respect to a reference point $D_{\text {ref }}$ on the highway. This reference point can either be embedded in DGPS maps or transmitted as a broadcast message by the fixed infrastructure nodes on the highway. We propose in Algorithm 1 a scheme for each vehicle to select its segment leader. As seen, each vehicle $v$ finds its current segment number according to the following:

$$
k=\left\lfloor\frac{D_{v}-D_{\mathrm{ref}}}{D_{c}}\right\rfloor .
$$

Based on the DGPS data, each vehicle can determine the end point of its current segment $D_{\text {end }}[k]$. Together with its own position $D_{v}$ and speed $S_{v}$, each vehicle can also determine the remaining time that it will stay within the current segment $T_{v \text {,rem }}$. Information regarding the positions and speeds of other vehicles in the same segment is received via the periodic safety messages. With this information, every vehicle can also calculate the remaining time that other vehicles stay within their respective segments.

At the beginning, we select the segment leader vehicle as the one with the longest remaining time in its own segment, using the procedure FindSegmentLeader (). Note that the segment leader can be in any lane of a highway. Vehicles selected as the leaders will serve as the segment leaders for the entire duration that they are present in those segments. When a segment leader realizes that it will move to another segment within a certain time $T_{\exp }$ (its value can be taken as a multiple of synchronization interval $=100 \mathrm{~ms}$ ), this vehicle marks its status as 'Retired' for the remaining time of its presence in the current segment. This vehicle is also responsible for appointing a new segment leader, i.e., the one with the current highest remaining time in the segment. The retired segment leader notifies this appointment to the new segment leader and all other vehicles in the current segment.

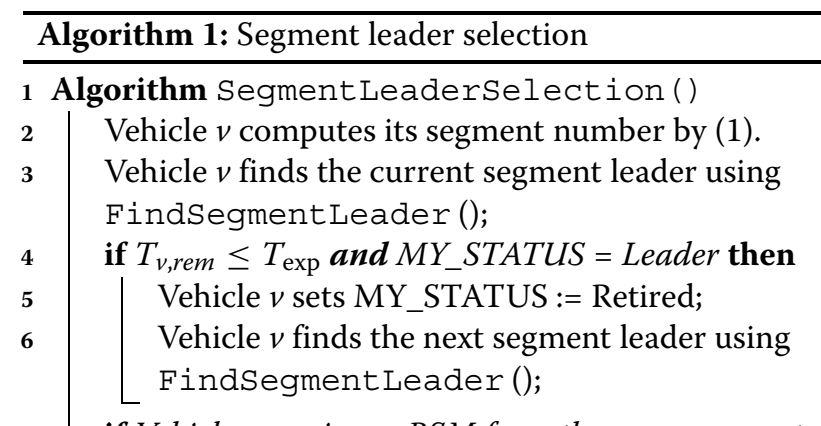

if Vehicle $v$ receives a BSM from the same segment with MY_STATUS = Retired and LEADER_ID equal to the node_ID of vehicle $v$ then

Vehicle $v$ sets MY_STATUS := Leader;

Vehicle $v$ sets $L E A D E R \_I D$ equal to the node_ID of vehicle $v$;

if Vehicle $v$ receives no BSM from the same segment with MY_STATUS $=$ Leader for a time period

$t>T_{\exp }$ then

11 Vehicle $v$ determines the segment leader using FindSegmentLeader ();

12 if Vehicle $v$ receives a BSM from the same segment with MY_STATUS = Leader or Vehicle $v$ moves to a new segment then

$13\lfloor$ Lehicle $v$ sets MY_STATUS := Regular;

14 Procedure FindSegmentLeader()

15 foreach Vehicle $w$ in the $k^{\text {th }}$ segment do

$16 \quad\left\lfloor T_{w, \text { rem }}=\left(D_{\text {end }}[k]-D_{w}\right) / S_{w}\right.$;

17 Vehicle $v$ selects segment leader of the $k^{\text {th }}$ segment as: $w^{*}=\arg \max _{w \in k^{t h}}$ segment $T_{w, \text { rem; }}$;

18 Vehicle $v$ sets its $L E A D E R \_I D$ field equal to the node_ID of $w^{*}$; 
To implement the above mechanism, we propose that every vehicle adds two new fields as part of its periodic safety messages. The MY_STATUS field indicates the current status of a vehicle, which can have values of 'Regular', 'Leader', and 'Retired'. The LEADER_ID field contains the node ID of the current segment leader. As described in Algorithm 1, when a vehicle receives a periodic safety message from a leader vehicle of the same segment (with MY_STATUS = Leader), the LEADER_ID field in that message contains the node ID of the leader.

When a segment leader retires, it finds the new segment leader and sends the safety message with the node ID of that new leader in the LEADER_ID field. Upon receiving the periodic safety message from the same segment with MY_STATUS = Retired, each vehicle matches its own node ID with the LEADER_ID field value. If a vehicle recognizes a match, it sets itself as the new leader and changes its MY_STATUS field to Leader.

A vehicle in a segment may not receive a periodic safety message from a leader vehicle of the same segment for a time $t>T_{\text {exp. }}$. In this case, that vehicle will find the leader using the procedure FindSegmentLeader(). Similarly, if a vehicle enters an empty segment, it will wait for $t=T_{\exp }$ before appointing itself as the leader of that segment. It may also happen that two vehicles in proximity both consider themselves as the leaders of the current segment. In this case, the vehicle first receiving the periodic safety message with MY_STATUS = Leader will change its MY_STATUS to Regular and become a 'Regular' vehicle. This arrangement guarantees that there will always be one single segment leader in any road segment.

In the proposed protocol, segment size is selected as a value much smaller than the safety message transmission range. This results in a high reception rate of safety messages and hence, reliable dissemination of leader information within a segment. Moreover, the segment size is taken as a fixed value as its a challenging task to adaptively vary and make consensus on the value of a segment size in an ad hoc network [35]. However, there are number of techniques found in the literature such as transmit power control and packet generation rate control that can be used to reduce the safety message traffic within a segment at higher vehicle densities $[8,17,36,37]$. This nonetheless, is out of the scope of this paper which is focused on efficient multi-hop message dissemination. Finally, it should be noted that if a segment could not elect a segment leader for a certain time period during which the warning message is also disseminated, that segment will not be part of relay selection and will not effect the working of protocol as will be discussed in Section 3.3.

\subsection{Multi-hop time-slot reservation mechanism}

We propose a multi-hop broadcast protocol using a timeslot reservation mechanism in Algorithm 2. Note that safety messages are uniformly generated within a synchronization interval of $100 \mathrm{~ms}$, and they employ the carrier-sense multiple access with collision avoidance (CSMA/CA) mechanism [16]. Here, we propose to use separate time slots for multi-hop warning message transmissions, each of which has the structure as depicted in Figure 2. Time is divided into continuous multi-hop time slots which are only reserved in the presence of warning messages using the mechanism described below.

Let $T_{\text {mslot }}$ be the size of a multi-hop time slot. Referring to the time-slot structure of the proposed design in Figure 2, the multi-hop time slot size $T_{\text {mslot }}$ is computed as:

$$
T_{\text {mslot }}=T_{b}+T_{c}+T_{d}+T_{c n t}+T_{a}
$$

where $T_{b}$ is the black burst duration, $T_{c}$ is the CLEAR packet transmission time, $T_{d}$ is the DATA (warning message) transmission time, $T_{\text {cnt }}$ is the CONTENTION time, and $T_{a}$ is the ACK packet transmission time.

By knowing the value of $T_{\text {mslot }}$ and current time using the DGPS, each vehicle finds the start time of the next multi-hop time slot $T_{\text {mhop }}[k]$. Vehicles with a queued

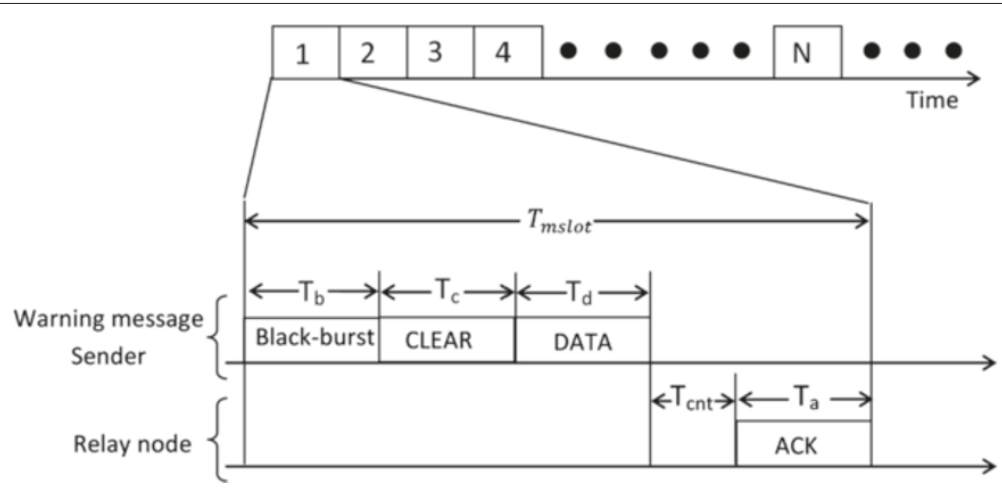

Figure 2 Structure of a time slot in the proposed multi-hop broadcast protocol. 


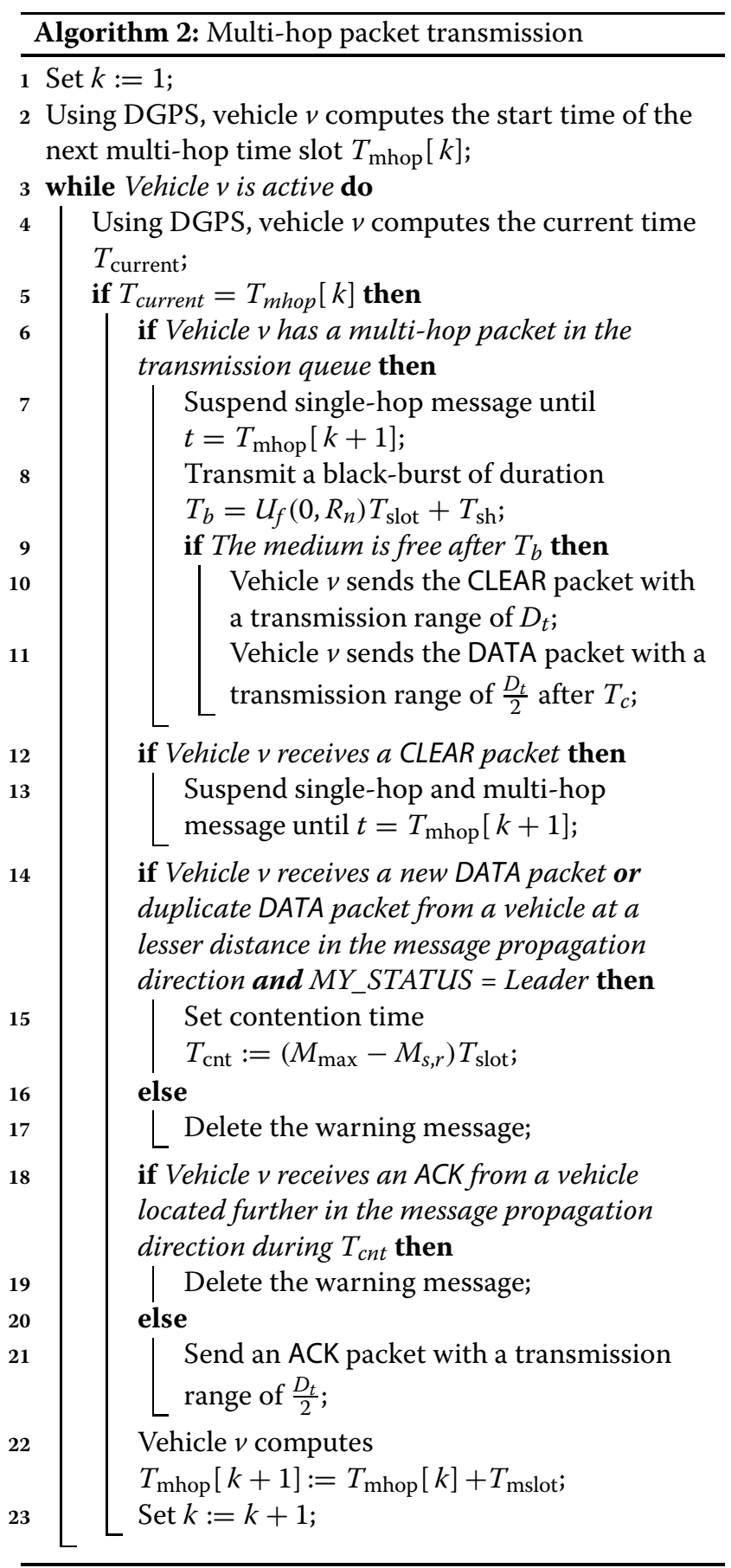

multi-hop packet suspends transmitting any single-hop message generated during the current multi-hop time slot until the start of the next multi-hop time slot $T_{\text {mhop }}[k+1]$. Specifically, the proposed multi-hop time slot starts with the transmission of a black burst energy signal (channel jamming signal) $[23,38]$ of duration $T_{b}=U_{f}\left(0, R_{n}\right) T_{\text {slot }}+$ $T_{\mathrm{sh}}$, where $U_{f}(x, y)$ represents a uniformly distributed random value in the interval $[x, y], R_{n}$ is an integer, $T_{\text {slot }}$ is the slot time in the IEEE 802.11 standard, and $T_{\mathrm{sh}}$ is the single-hop message transmission time. The rationale behind transmission of the black burst signal is to temporarily suspend the ongoing single-hop messages. By implementing the black burst signaling, the CLEAR packet used for multi-hop time slot reservation is not interfered by the single-hop messages. The length of the black burst signal is at least equal to the transmission time of a singlehop message to allow all single-hop messages transmitted before the black burst to complete their transmissions. Each vehicle with a queued multi-hop message picks a random integer in the range $\left[0, R_{n}\right]$ to calculate the duration of its black burst signal. Upon detecting the black burst energy signal, vehicles within transmission range $D_{t}$ suspend their single-hop messages because the medium is busy.

The vehicle with the longest black burst signal will find the medium free after the $T_{b}$ period and then transmit a CLEAR packet at a transmission range $D_{t}$. The purpose of the CLEAR packet is to reserve the rest of the time slot for the multi-hop warning message, and also to inform the vehicles in the range $D_{t}$ of the upcoming multi-hop warning message transmission. Upon receiving the CLEAR packet, vehicles suspend their queued multi-hop warning message until the start of the next multi-hop time slot $T_{\text {mhop }}[k+1]$. Also, vehicles with a single-hop safety message generated during the current multi-hop time slot suspend their transmission until the start of the next multi-hop time slot $T_{\text {mhop }}[k+1]$. As such, all vehicles in the range $D_{t}$ who intend to send periodic safety messages during the reserved multi-hop time slot will not interfere with the multi-hop warning-message transmission. Vehicles who do not receive a CLEAR packet may continue transmitting its safety message during the multi-hop time slot. This arrangement allows full utilization of the multi-hop time slot in the absence of an emergency warning message.

After the CLEAR phase, the vehicle who has a reserved multi-hop time slot is allowed to send the warning message (i.e., DATA) at a transmission range of $D_{t} / 2$. Since the CLEAR packet is transmitted over a range of $D_{t}$, all hidden nodes located within two transmission hops from the warning-message sender are made aware of the upcoming warning message transmission. Essentially, the interference from any hidden nodes during the transmission of such a message is eliminated.

In the CONTENTION phase, each segment leader first checks if it is a new warning message. If a duplicate DATA packet is received from a vehicle located further in the message direction, the warning message is deleted as the message has already progressed in the propagation direction. Otherwise, a contention time $T_{\text {cnt }}$ is calculated by the segment leader. We propose that the value of $T_{\mathrm{cnt}}$ is inversely proportional to the segment separation between 
the sender of the warning message and the segment leader (i.e., the receiver):

$$
T_{\text {cnt }}=\left(M_{\max }-M_{s, r}\right) T_{\text {slot }}
$$

where $M_{\max }$ is the maximum number of segments within the warning message transmission range, $M_{s, r}$ is the segment separation between the sender and the receiver, and $T_{\text {slot }}$ is the slot time.

In the ACK phase, the segment leader with the shortest contention time $T_{\mathrm{cnt}}$ will transmit an ACK packet. It is worth recalling that this leader is responsible for relaying the warning message in the next time slot. After receiving the ACK of the warning message from the winning leader in the current time slot, all other segment leaders (i.e., potential forwarders) delete the corresponding warning messages in their queues. No other actions are required from these vehicles. Note that such a message cancellation policy only applies when a segment leader receives an ACK from a vehicle located further away in the direction of message propagation. This ensures the progress of the warning message in the propagation direction. It might also happen that the vehicle who has forwarded the warning message does not receive an ACK within the current time slot. In such a case, this vehicle will resend the same warning message in one of the next time slots that are reserved for multi-hop warning message dissemination.

\subsection{Handling the lost ACK scenarios}

It is possible that the ACK packet sent by the segment leader responsible for relaying the warning message might get lost during the transmission. In the absence of an ACK, other segment leaders would have to resend the warning message unnecessarily. While the exact number of redundant retransmissions depends on how many vehicles have received the ACK packet, it can be substantial in poor channel conditions. In this paper, we propose the following arrangements to handle the ACK loss situation and thereby improving the efficiency of channel utilization.

Illustrated in Figure 3 is a warning message transmission scenario, where all 7 vehicles are the leaders in their

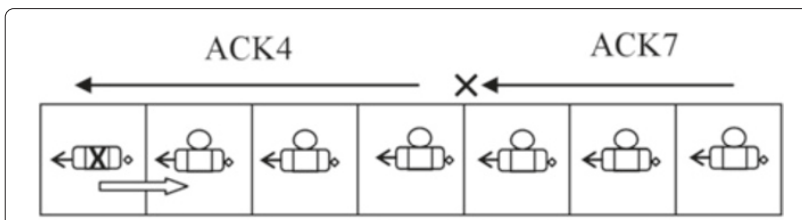

$\begin{array}{lllllll}\text { Seg.1 } & \text { Seg.2 } & \text { Seg.3 } & \text { Seg.4 } & \text { Seg.5 } & \text { Seg.6 } & \text { Seg.7 }\end{array}$

Figure 3 Lost ACK scenario. respective road segments. Suppose that the warning message sent by vehicle 1 is received by vehicles 2 to 7 , as the furthest leader from vehicle 1 , vehicle 7 has its contention timer expired first as determine by (3) and thus sends an ACK7 packet. Suppose that this ACK7 packet is not received by all other vehicles, e.g., due to severe interference or deep channel fading, only vehicles 5 and

Table 1 Simulation parameters

\begin{tabular}{|c|c|c|}
\hline \multicolumn{2}{|c|}{ Parameter } & \multirow{2}{*}{$\begin{array}{l}\text { Value } \\
2,4,6 \mathrm{~km} \\
\end{array}$} \\
\hline \multirow{4}{*}{ Highway Road } & Road length & \\
\hline & Number lanes & 6 (3 per direction) \\
\hline & Segment size & $75 \mathrm{~m}$ \\
\hline & $\begin{array}{l}\text { Maximum number of } \\
\text { segment } M_{\max }\end{array}$ & 6 \\
\hline \multirow{3}{*}{ Vehicle } & Medium density & 120 (vehicles/km) \\
\hline & High density & 180,240 (vehicles $/ \mathrm{km}$ ) \\
\hline & Speed & $60,45,30(\mathrm{~km} / \mathrm{h})$ \\
\hline \multirow{5}{*}{ Safety message } & Size & 500 bytes \\
\hline & Data rate & $6 \mathrm{Mbps}$ \\
\hline & Transmission range & $300 \mathrm{~m}$ \\
\hline & Generation frequency & $10 \mathrm{~Hz}$ \\
\hline & $T_{\text {sh }}$ & $0.71 \mathrm{~ms}$ \\
\hline \multirow[t]{3}{*}{ Warning message } & $\begin{array}{l}\text { Number of } \\
\text { warning nodes }\end{array}$ & $1,3,5$ \\
\hline & $\begin{array}{l}\text { Warning notification } \\
\text { region }\end{array}$ & $2 \mathrm{~km}$ \\
\hline & Generation frequency & $2 \mathrm{~Hz}$ \\
\hline \multirow{3}{*}{ Black burst signal } & $R_{n}$ & 7 \\
\hline & Slot time $T_{\text {slot }}$ & $13 \mu \mathrm{s}$ \\
\hline & $T_{b}$ & $0.81 \mathrm{~ms}$ \\
\hline \multirow{3}{*}{ CLEAR packet } & Size & 8 bytes \\
\hline & $T_{c}$ & $0.055 \mathrm{~ms}$ \\
\hline & Transmission range & $1,000 \mathrm{~m}$ \\
\hline \multirow{3}{*}{ DATA message } & Size & 500 bytes \\
\hline & $T_{d}$ & $0.71 \mathrm{~ms}$ \\
\hline & Transmission range & $500 \mathrm{~m}$ \\
\hline \multirow{9}{*}{ ACK packet } & Size & 38 bytes \\
\hline & $T_{a}$ & $0.095 \mathrm{~ms}$ \\
\hline & Transmission range & $500 \mathrm{~m}$ \\
\hline & $\begin{array}{l}\text { Multi-hop time } \\
\text { slot size, } T_{\text {mslot }}\end{array}$ & $1.75 \mathrm{~ms}$ \\
\hline & $T_{\exp }$ & $0.5 \mathrm{~s}$ \\
\hline & Fading model & Nakagami- $m(m=1,3,5)$ \\
\hline & Reception $R x_{\text {th }}$ & $-91 \mathrm{dBm}$ \\
\hline & Background noise & $-99 \mathrm{dBm}$ \\
\hline & Simulation time & $300 \mathrm{~s}$ \\
\hline
\end{tabular}




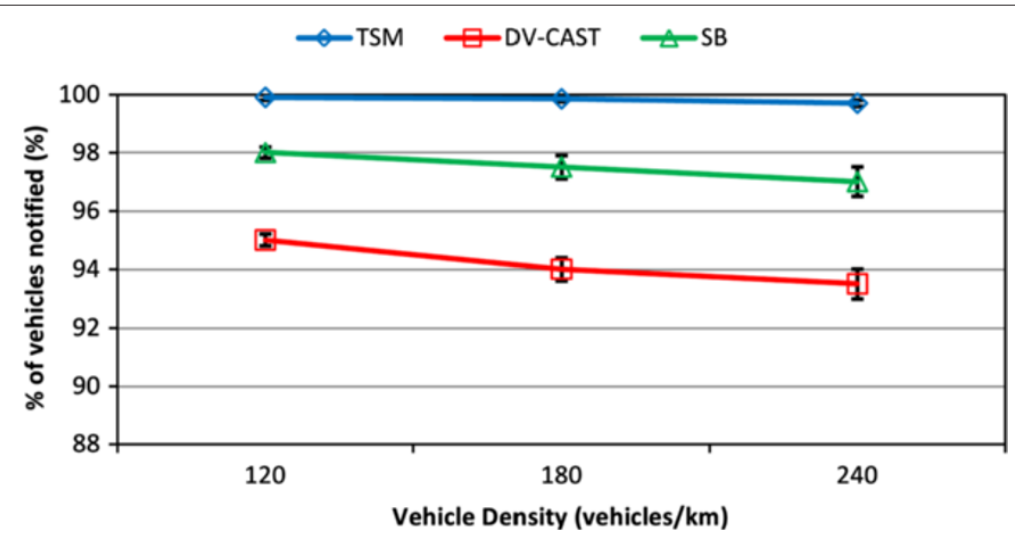

Figure 4 Reception rate of warning messages at different vehicle densities for $L_{r}=2, X=3$, and $m=3 \mathrm{~km}$.

6 receive this $A C K 7$ packet. Without any ACK7 reception, vehicle 4 assumes that it is responsible for relaying the warning message and thus sends an ACK4 packet after the CONTENTION phase.

In the next time slot, both vehicles 4 and 7 will have the warning messages (originally from vehicle 1 ) in their queues to be relayed to further nodes. If vehicle 7 wins in the CONTENTION phase of this time slot, it will broadcast the warning message. In this case, vehicle 4 will receive a duplicate data packet from a vehicle located further away in the direction of message propagation (i.e., vehicle 7). Knowing that the warning message has progressed further, vehicle 4 can just delete the warning message in its queue and take no further action. On the other hand, if vehicle 4 wins in the CONTENTION phase, it will broadcast this warning message. In this case, vehicle 7 will receive a duplicate data packet from a vehicle at a lesser distance in the direction of message propagation (i.e., vehicle 4). Vehicle 7 will then reply with an ACK7 packet to notify vehicle 4 that the warning message has progressed further, and no other action is required from vehicle 4 . In either scenario, the unnecessary retransmissions of the warning message are avoided.

\section{Performance evaluation}

The performance of the proposed time-slotted multi-hop transmission (TSM) protocol is evaluated in this section using an OPNET Modeler 16.0 simulation model. Specifically, we consider a highway scenario of road length $L_{r}$ assuming 3 lanes in each direction of the road. At medium vehicle density (120 vehicles $/ \mathrm{km})$, we assume an exponentially distributed inter-vehicle spacing; whereas at high vehicle densities (180 and 240 vehicles $/ \mathrm{km}$ ), we assume normally distributed inter-vehicle spacing as specified in reference [39]. The propagation model used for highway VANET simulation scenario in this paper is the free space path loss with a path loss exponent of $1.8[40,41]$. The average received power using this propagation model at a distance $d$ is given as:

$$
P_{r}(d)=\frac{P_{t} G_{t} G_{r} \lambda^{2}}{(4 \pi)^{2} d^{1.8}}
$$

where $P_{t}$ is the transmitted power, $G_{t}$ is the transmitter antenna gain, $G_{r}$ is the receiver antenna gain, and $\lambda$ is the carrier wave length. Using receiver threshold $R x_{\text {th }}$ and (4), we calculate the transmission range of CLEAR,

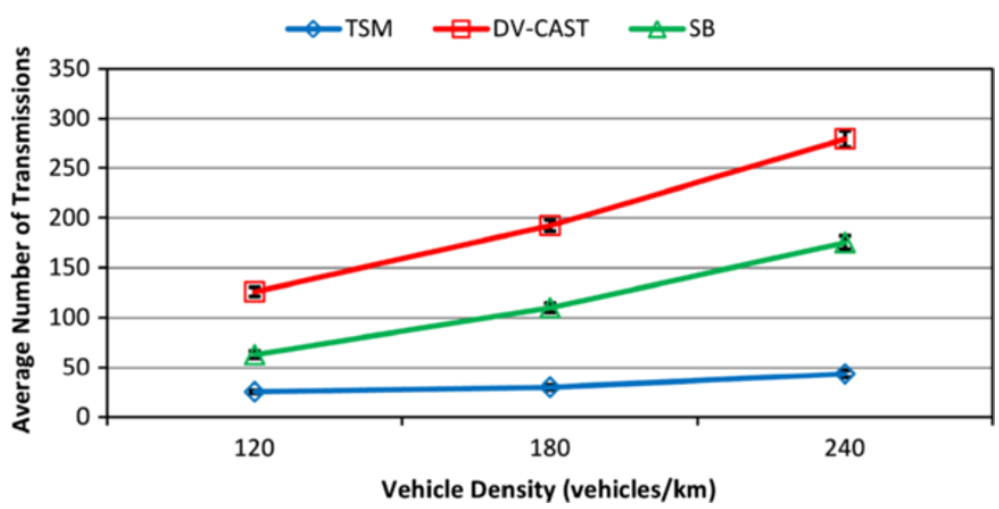

Figure 5 Average number of warning transmissions at different vehicle densities for $L_{r}=2, X=3$, and $m=3 \mathrm{~km}$. 


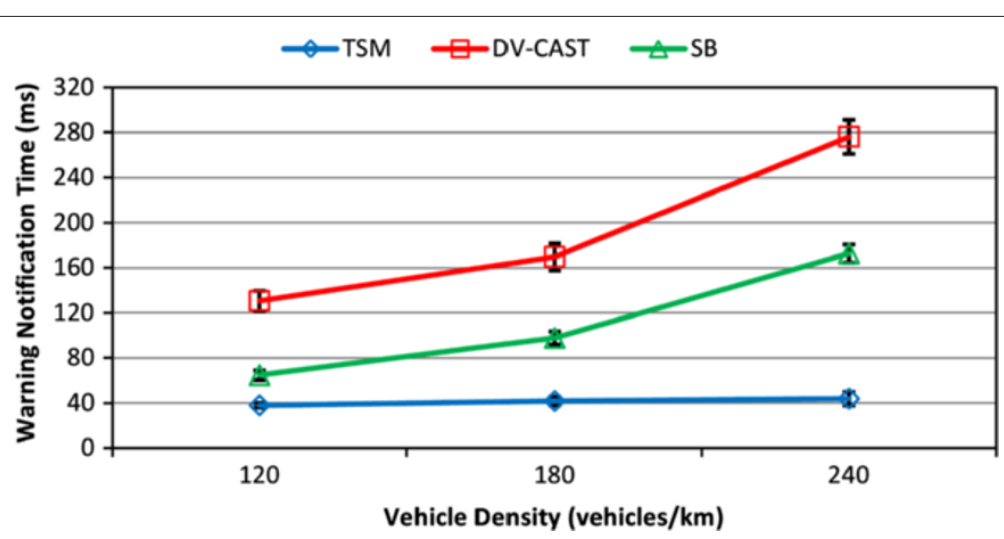

Figure 6 Warning notification time at different vehicle densities for $L_{r}=2, X=3$, and $m=3 \mathrm{~km}$.

DATA, and ACK messages in Figure 1. To model fading in vehicular environments, we use Nakagami- $m$ fading with different values of fading intensity $m$ as specified for mobile-to-mobile vehicular propagation channels $[42,43]$.

For the warning messages, we place $X$ equally spaced emergency warning vehicles within the length $L_{r}$ of the road section. Each of these vehicles generates a different warning message to be propagated in the entire road section. Unless otherwise mentioned, $L_{r}, X$, and $m$ are taken as 2, 3, and 3, respectively. We also study the effect of varying these parameters on the warning message performance in the simulations.

Every vehicle also generates periodic safety messages with a transmission range of $300 \mathrm{~m}$ at $10 \mathrm{~Hz}$ frequency as specified for most safety applications $[5,44]$. A CLEAR packet is sent with the maximum transmission range of 1,000 m specified in the IEEE 802.11p standard [16]. Since the CLEAR packet informs vehicles in the two-hop transmission range of an upcoming warning message, the DATA (warning message) and the ACK packets are assigned a transmission range of $500 \mathrm{~m}$. The key parameters used in our simulations are listed in Table 1. Each simulation is run for $300 \mathrm{~s}$, and the simulation results are plotted with 95\% confidence intervals.

For the multi-hop time slot parameters, $R_{n}$ is selected as 7 and $T_{\text {slot }}$ is $13 \mu \mathrm{s}$ in duration. The contention time $T_{\text {cnt }}$ is determined by (3), where the maximum segment size $M_{\max }=6$ is used for the $500 \mathrm{~m}$ transmission range and the $75 \mathrm{~m}$ road segment size. The size of CLEAR, DATA and ACK packets used are 8,500 and 38 bytes respectively. From (2), $T_{\text {mslot }}=1.75 \mathrm{~ms}$.

We compare our proposed TSM transmission design with two existing widely cited protocols selected from each category of multi-hop protocols as mentioned in Sec. 2, namely, the DV-CAST [12] and the SB [20]. The DV-CAST uses a distance based contention mechanism whereas the SB protocol employs a similar segment division based multi-hop transmission technique. In the simulation study, the multi-hop transmission range, the number of time slots, and the maximum wait time for the DV-CAST protocol are taken as 500, 5, and $5 \mathrm{~ms}$, respectively [12]. On the other hand, the multi-hop transmission range and the number of sectors for the SB protocol are set as 500 and $20 \mathrm{~m}$, respectively [20].

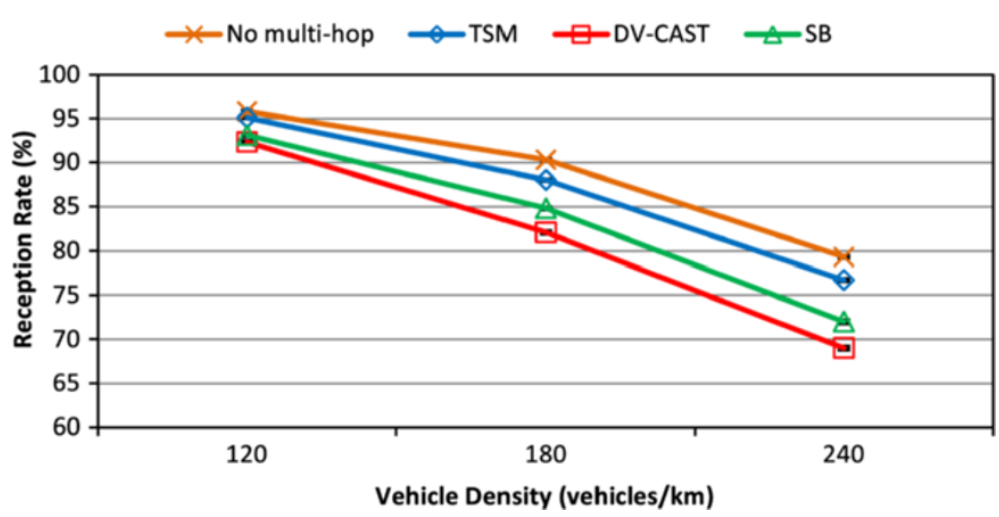

Figure 7 Reception rate of safety messages at different vehicle densities for $L_{r}=2, X=3$, and $m=3 \mathrm{~km}$. 


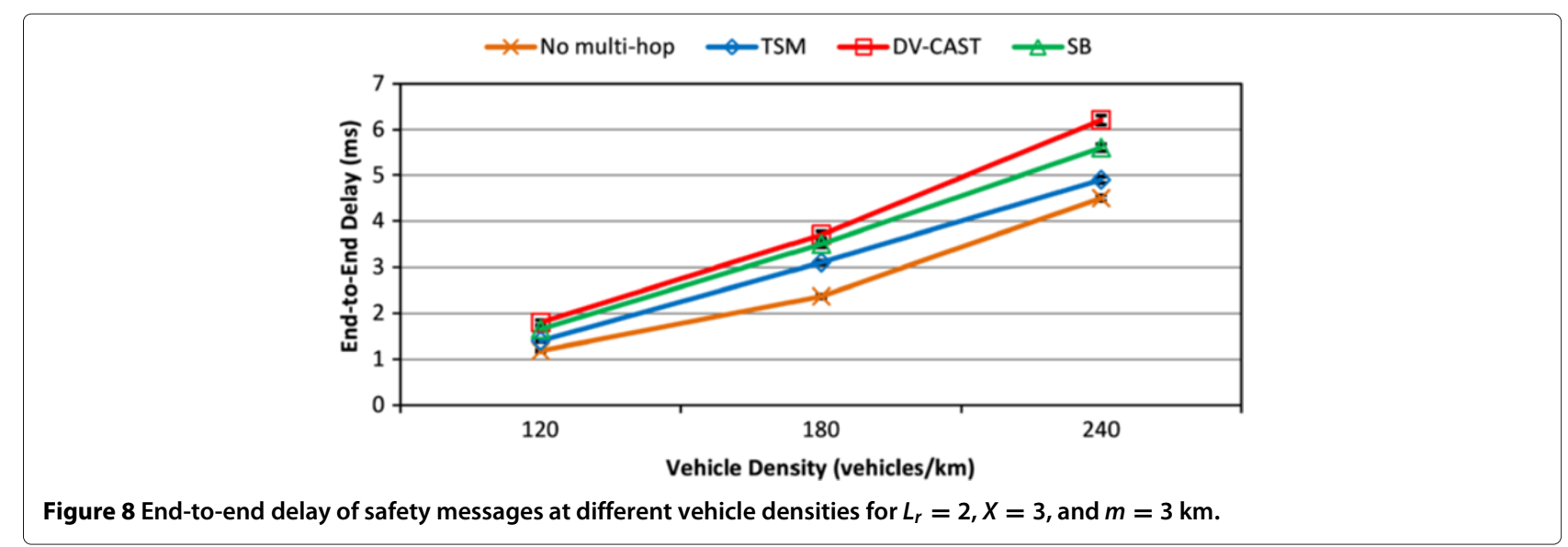

\subsection{Performance metrics}

Following performance metrics are used to evaluate the performance of multi-hop warning and single-hop safety messages.

- Reception rate of warning messages: Proportion of vehicles that successfully receive a transmitted warning message.

- Average number of warning transmissions: Average number of warning message transmissions required to successfully disseminate all $X$ warning messages.

- Warning notification time: Average time period required to inform all the vehicles within the road section of the $X$ warning messages.

- Reception rate of safety messages: Packet success rate of safety messages within a distance of $100 \mathrm{~m}$ between the transmitter and the receiver.

- End-to-end delay of safety messages: Average time period required to transmit a safety message from the source to the destination.

- Medium busy percentage: Percentage of simulation time the medium is sensed as busy by the vehicles due to receiving a signal greater than the carrier sense threshold [17].

In the following, we present four different scenarios that evaluate the effect of varying vehicle density, road length, number of warning message senders, and channel conditions on the warning message performance. While it is possible to evaluate all the metrics in each scenario, we only present the important metrics to keep the discussion concise.

\subsection{Effect of varying vehicle density}

In this subsection, we evaluate the abovementioned performance metrics at different vehicle densities. The values of $L_{r}, X$, and $m$ are taken as 2,3 , and $3 \mathrm{~km}$, respectively. In Figure 4, we plot the reception rate of the warning messages. As can be seen, our proposed solution guarantees an almost $100 \%$ delivery rate for every vehicle density scenario considered. This is a noticeable enhancement in light of the $93 \%$ to $98 \%$ reception rates provided by the DV-CAST and the SB protocols. The proposed TSM protocol uses an explicit acknowledgment from a vehicle further in the direction of message propagation as

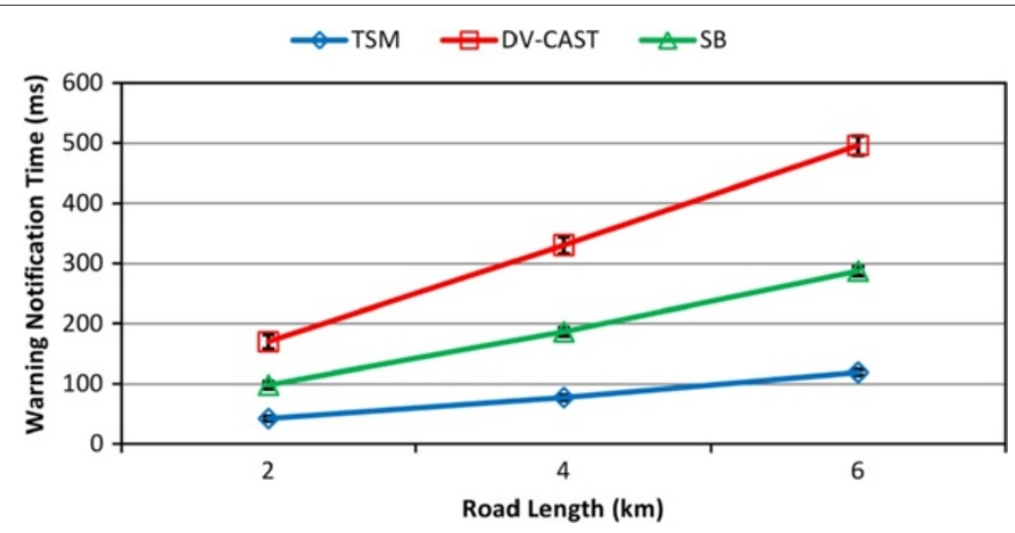

Figure 9 Warning notification time at different road lengths for $\rho=180, X=3$, and $m=3$ vehicles $/ \mathrm{km}$. 


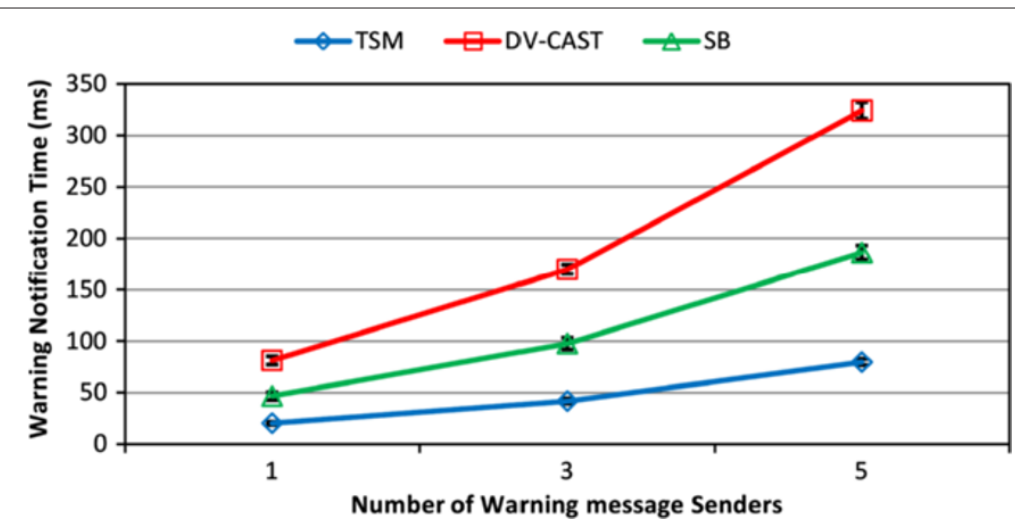

Figure 10 Warning notification time at different number of warning message transmitters for $\rho=180$ vehicles $/ \mathrm{km}, L_{r}=2 \mathrm{~km}$, and $m=3$.

a message cancellation policy. On the other hand, both the DV-CAST and the SB protocols cancel the warning message forwarding once a duplicate is received from either direction. This halts the progress of a warning notification when the implicit acknowledgment (in case of DV-CAST)/ clear to broadcast packet (in case of SB) from a vehicle nearer to the source cancels the message forwarding in the message propagation direction. Due to this reason, both protocols show a reception rate of less than $100 \%$.

We display in Figure 5 the average number of warning transmissions. In comparison to the DV-CAST and the SB protocols, our proposed TSM scheme significantly reduces the number of required transmissions. Particularly, at the vehicle density of 240 vehicles $/ \mathrm{km}$, only 44 transmissions are needed for the three warning messages. This figure represents a mere $10 \%$ to $15 \%$ of the total number of transmissions required by the existing approaches. Such a remarkable gain is a direct result of the novel features of our design: (i) the suppression of the broadcast storm by only allowing segment leaders to act as the potential forwarders, (ii) the interference avoidance and the hidden node resolution by employing the time-slotted structure and the CLEAR packet, and (iii) the arrangement to actively handle the ACK packet loss scenarios.

Figure 6 shows the warning notification time of the three protocols. It is clear from the figure that the proposed TSM protocol has a much lower dissemination time compared to both the DV-CAST and the SB protocols. This is due to less number of transmissions required by the TSM protocol as shown in Figure 5, which results in a quicker progress of the warning message. In particular, at a vehicle density of 120 vehicles $/ \mathrm{km}$, the TSM notification time is 92 and 26 ms shorter than the DVCAST and the SB counterparts. As the number of vehicles per $\mathrm{km}$ increases, the notification time incurred by the DV-CAST and the SB schemes significantly grows due to higher interference from the periodic safety messages. However, the effect of interference is limited in case of the proposed TSM protocol since it employs separate time slots to send the warning messages. From Figure 6, it is evident that the resulting TSM notification time only slightly increases while remaining below $45 \mathrm{~ms}$ for all the

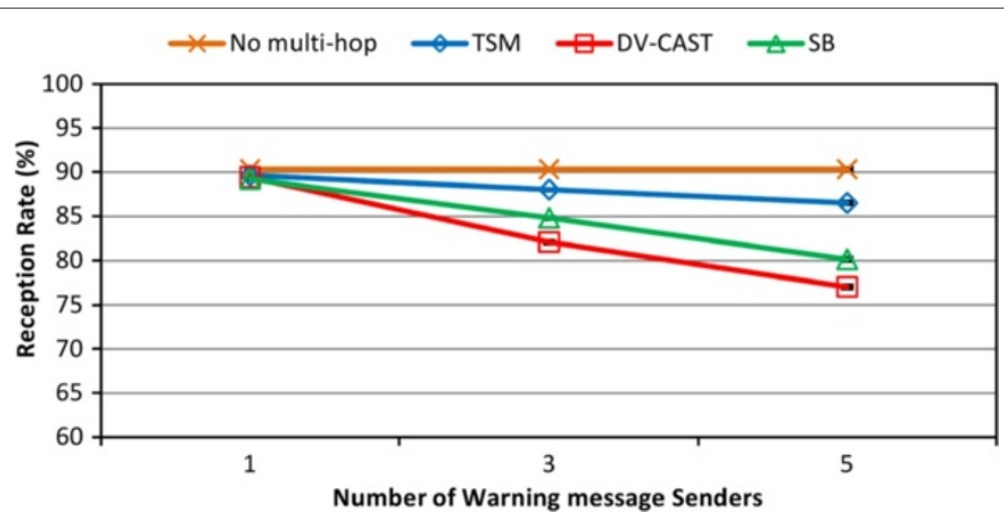

Figure 11 Reception rate of safety messages at different number of warning message transmitters for $\rho=180$ vehicles $/ \mathrm{km}, L_{r}=2 \mathrm{~km}$ and $m=3$. 


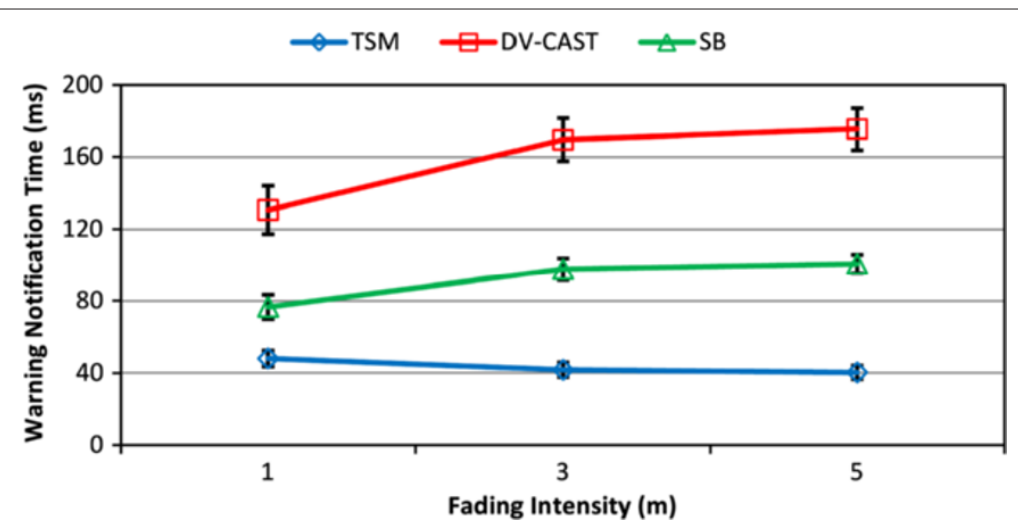

Figure 12 Warning notification time at different fading intensities for $\rho=180$ vehicles $/ \mathrm{km}, L_{r}=2 \mathrm{~km}$, and $X=3$.

vehicle densities under consideration. Finally, the most pronounced advantage is observed at the vehicle density of 240 vehicles $/ \mathrm{km}$, where the warning notification time of the proposed TSM protocol is dropped to 230 and $130 \mathrm{~ms}$ compared to the DV-CAST and the SB protocols respectively.

We also examine the effects of our proposed multi-hop warning message transmission protocol on the existing periodic single-hop safety messages. It is apparent from Figure 7 that while the reception rate of the safety messages is degraded in presence of warning messages, the effect is mild in the TSM protocol. At the density of 240 vehicles $/ \mathrm{km}$, the loss of safety messages caused by the proposed TSM warning message transmissions is $3 \%$ higher than the scenario when no warning messages are transmitted. This is a clear improvement from the $10 \%$ and $7 \%$ losses incurred by the DV-CAST and the SB protocols. A similar trend can also be observed from Figure 8, albeit in terms of the end-to-end delay of safety messages. Here, the TSM solution offers a $10 \%$ to $20 \%$ reduction in the end-to-end delay, while following quite closely with the delay in the case of no warning message transmissions.

\subsection{Effect of varying road length}

To evaluate the effect of road length, we plot the warning notification time at a vehicle density of 180 vehicles $/ \mathrm{km}$ in Figure 9. The values of $X$ and $m$ are selected as 3 each. As the road length is increased, the warning message needs to travel a larger distance using greater number of hops. As a result, the warning notification time of all protocols show a rise. It can be seen that the TSM protocol shows a much better performance at longer road lengths due to its segment leader-based relay selection and separate time slot allocation for warning messages. Particularly, at a road length of $6 \mathrm{~km}$, the warning notification time of the TSM, the $\mathrm{SB}$, and the DV-CAST protocols is 119, 287, and 496 $\mathrm{ms}$, respectively.

\subsection{Effect of varying number of warning message senders} Varying the number of warning message senders $X$, Figure 10 shows the warning notification time at a vehicle density of 180 vehicles $/ \mathrm{km}$. The values of $L_{r}$ and $m$ are selected as 2 and $3 \mathrm{~km}$, respectively. In the presence of a single warning message sender, the TSM protocol shows 26 and $60 \mathrm{~ms}$ improvement over the SB and the DVCAST protocols, respectively. However, increase in the

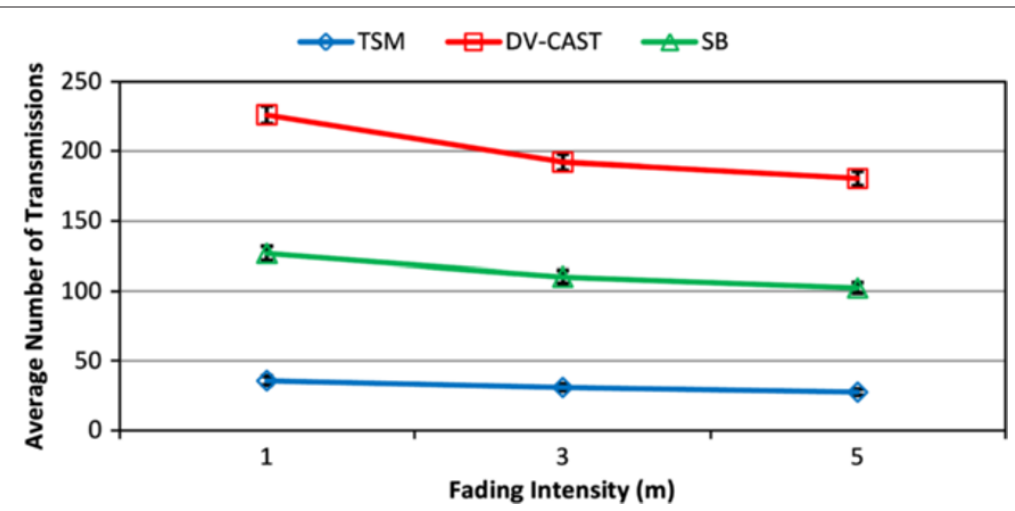

Figure 13 Average number of warning transmissions at different fading intensities for $\rho=180$ vehicles $/ \mathrm{km}, L_{r}=2 \mathrm{~km}$, and $X=3$. 


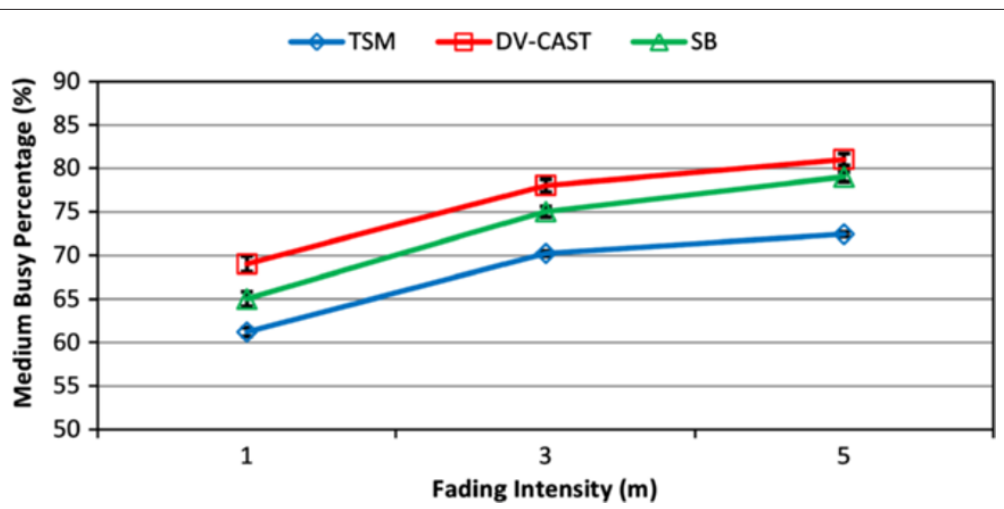

Figure 14 Medium busy percentage at different fading intensities for $\rho=180$ vehicles $/ \mathrm{km}, L_{r}=2 \mathrm{~km}$, and $X=3$.

number of warning message senders results in a greater interference with the safety messages; hence clearly showing the significance of the proposed TSM protocol. The TSM protocol that uses black burst and CLEAR to reserve a multi-hop time slot along with the relay selection using segment leaders mitigates the interference between the safety and the warning messages. Specifically, the TSM protocol has 106 and $244 \mathrm{~ms}$ lower warning notification time as compared to the SB and the DV-CAST protocols, respectively, when the number of warning message senders increase to 5 .

In Figure 11, the reception rate of safety messages is presented. As the number of the warning messages on the road increases, reception rate of safety message goes down due to interference. It can be seen from the result that the TSM protocol provides a much better performance against interference due to its separate time slot allocation mechanism. Particularly, reception rate of safety messages is improved by $6 \%$ to $9 \%$ when the number of warning message senders increase to 5 .

\subsection{Effect of varying channel conditions}

In this subsection, we evaluate the effect of varying channel conditions on the performance of warning and safety messages. The values of $L_{r}$ and $X$ are taken as 2 and 3 $\mathrm{km}$. Note that a lower value of $m$ represents more severe fading. For the DV-CAST and the SB protocols, the warning notification time reduces as the $m$ value goes lower depicted in Figure 12. Although the number of transmissions increase at higher values of fading (lower $m$ ) because of the poor channel conditions as shown in Figure 13, the reduction in notification time can be explained as follows. At lower value of $m$, medium busy percentage is significantly reduced as shown in Figure 14. This is due to higher packet losses at the lower value of $m$ causing reduction in reception rate of safety messages as shown in Figure 15. As a result, warning messages are transmitted quicker due to less interference from the safety messages, in spite of the increased number of warning transmissions. Therefore, a higher fading improves the warning notification time of the SB and the DV-CAST protocols.

On the other hand, warning notification time for the TSM protocol shown in Figure 12 decreases by $10 \mathrm{~ms}$ as $m$ is increased from 1 to 3 . Also, the average number of warning transmissions plotted in Figure 13 decreases as $m$ is increased due to improved channel conditions. Since the TSM protocol uses separate multi-hop time slots for warning message dissemination, the decrease in

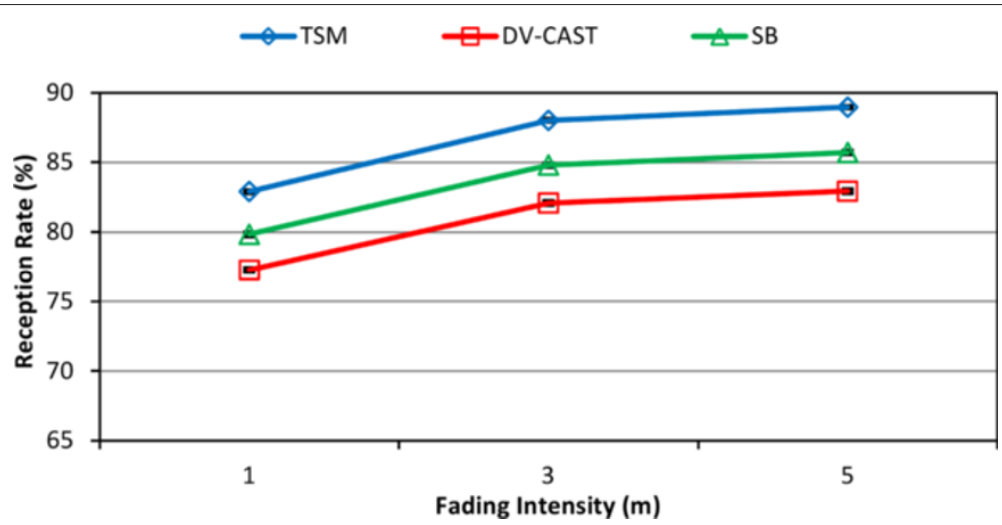

Figure 15 Reception rate of safety messages at different fading intensities for $\rho=180$ vehicles $/ \mathrm{km}, L_{r}=2 \mathrm{~km}$, and $X=3$. 
medium busy percentage and safety message reception rate at lower value of $m$ does not effect the warning transmissions. The result shows that the TSM protocol is more resilient against fading maintaining nearly constant warning notification time (less than $50 \mathrm{~ms}$ ) as the channel conditions vary. This is an improvement of 28 to $82 \mathrm{~ms}$ over the $\mathrm{SB}$ and the DV-CAST protocols at $m=1$.

\section{Conclusions}

In this paper, we have presented a multi-hop broadcast protocol to efficiently disseminate emergency warning messages in a VANET. To address the broadcast storm problem, we have adopted the approach of road segment division and selected the segment leaders who are responsible for relaying the warning messages over multiple hops to further nodes. To reduce the mutual interference with the existing periodic safety messages, we have proposed a time-slotted structure for the multi-hop transmissions. Specifically, we have assigned separate time slots for the warning messages and proposed the use of a black burst signal and a CLEAR packet before the actual data transmission to eliminate all the hidden nodes in range and reserve the multi-hop time slot. To avoid the unnecessary retransmissions of the warning messages, we have established a mechanism that effectively handles the case of ACK packet losses. Simulation results with realistic parameters have verified the clear advantages of our proposed scheme over existing solutions in several key performance criteria.

\section{Competing interests}

The authors declare that they have no competing interests.

Received: 23 July 2014 Accepted: 17 October 2014

Published: 30 October 2014

\section{References}

1. R Chen, W-L Jin, A Regan, Broadcasting safety information in vehicular networks: issues and approaches. IEEE Network. 24(1), 20-25 (2010)

2. YToor, P Muhlethaler, A Laouiti, Vehicle ad hoc networks: applications and related technical issues. IEEE Commun. Surv. Tutor. 10(3), 74-88 (2008)

3. J Wan, D Zhang, S Zhao, L Yang, J Lloret, Context-aware vehicular cyber-physical systems with cloud support: architecture, challenges, and solutions. IEEE Commun. Mag. 52(8), 106-113 (2014)

4. D Caveney, Cooperative vehicular safety applications. IEEE Control Syst. 30(4), 38-53 (2010)

5. The European Telecommunications Standards Institute: ETSI TS 102 637-2 v1.2.1 - Intelligent transport systems (ITS) - Vehicular communications Basic set of applications - Part2: Specification of cooperative awareness basic service. Technical report (ETSI, Sophia Antipolis Cedex, France, 2011)

6. R Stanica, E Chaput, A-L Beylot, Properties of the MAC layer in safety vehicular ad hoc networks. IEEE Commun. Mag. 50(5), 192-200 (2012)

7. X Ma, J Zhang, X Yin, KS Trivedi, Design and analysis of a robust broadcast scheme for VANET safety-related services. IEEE Trans. Vehic. Technol. 61(1), 46-61 (2012)

8. C-L Huang, YP Fallah, R Sengupta, H Krishnan, Adaptive intervehicle communication control for cooperative safety systems. IEEE Network. 24(1), 6-13 (2010)

9. J Sahoo, Wu EH-K, PK Sahu, M Gerla, Congestion-controlled-coordinatorbased MAC for safety-critical message transmission in VANETs. IEEE Trans. Intell. Transportation Syst. 14(3), 1423-1437 (2013)
10. J Sahoo, EH-K Wu, PK Sahu, M Gerla, Binary-partition-assisted MAC-layer broadcast for emergency message dissemination in VANETs. IEEE Trans. Intell. Transportation Syst. 12(3), 757-770 (2011)

11. N Wisitpongphan, OK Tonguz, JS Parikh, P Mudalige, F Bai, V Sadekar, Broadcast storm mitigation techniques in vehicular ad hoc networks. IEEE Wireless Commun. 14(6), 84-94 (2007)

12. OK Tonguz, N Wisitpongphan, F Bai, DV-CAST: A distributed vehicular broadcast protocol for vehicular ad hoc networks. IEEE Wireless Commun. 17(2), 47-57 (2010)

13. M Torrent-Moreno, in Proc. Conf. on Wireless on Demand Network Systems and Services. Inter-vehicle communications: Assessing information dissemination under safety constraints (Oberguyrgl, Austria, 2007), pp. 59-64

14. M Li, K Zeng, W Lou, Opportunistic broadcast of event-driven warning messages in vehicular ad hoc networks with lossy links. Comput. Netw. 55(10), 2443-2464 (2011)

15. C Campolo, A Molinaro, Multichannel communications in vehicular ad hoc networks: a survey. IEEE Commun. Mag. 51(5), 158-169 (2013)

16. JB Kenney, Dedicated short-range communications (DSRC) standards in the United States. Proc. IEEE. 99(7), 1162-1182 (2011)

17. M Torrent-Moreno, J Mittag, P Santi, H Hartenstein, Vehicle-to-Vehicle communication: Fair transmit power control for safety-critical information. IEEE Trans. Vehic. Technol. 58(7), 3684-3703 (2009)

18. The Institute of Electrical and Electronics Engineers. IEEE Std 802.11-2007 (Revision of IEEE Std 802.11-1999), IEEE Standard for information technology - Telecommunications and information exchange between systems - Local and metropolitan area networks - Specific requirements - Part 11: Wireless LAN medium access control (MAC) and physical layer (PHY) specifications. Technical report (IEEE, New York, USA, December 2007)

19. MA Javed, JY Khan, DT Ngo, in Proc. IEEE Wireless Communication and Networking Conference. Joint space-division multiple access and adaptive rate control for basic safety messages in VANETs (Istanbul, Turkey, 2014), pp. 1-5

20. E Fasolo, A Zanella, M Zorzi, in Proc. IEEE Intl. Conf. on Communications. An effective broadcast scheme for alert message propagation in vehicular ad hoc networks (Istanbul, Turkey, 2006), pp. 3960-3965

21. RS Schwartz, RRR Barbosa, N Meratnia, G Heijenk, H Scholten, A directional data dissemination protocol for vehicular environments. Comput. Commun. 34(17), 2057-2071 (2011)

22. M Slavik, I Mahgoub, Spatial distribution and channel quality adaptive protocol for multihop wireless broadcast routing in VANET. IEEE Trans. Mobile Comput. 12(4), 722-734 (2013)

23. G Korkmaz, E Ekici, F Özgüner, U Özgüner, in Proc. ACM Intl. Workshop on Vehicular Adhoc Networks. Urban multi-hop broadcast protocol for inter-vehicle communication systems (USA, Philadelphia, 2004), pp. 76-85

24. M Li, Z Li, A Vasilakos, A survey on topology control in wireless sensor networks: taxonomy, comparative study, and open issues. Proc. IEEE. 101(12), 2538-2557 (2013)

25. Y Yao, Q Cao, A Vasilakos, in Proc. IEEE Intl. Conf. on Mobile Ad-Hoc and Sensor Systems. Edal: An energy-efficient, delay-aware, and lifetime-balancing data collection protocol for wireless sensor networks, (2013), pp. 182-190

26. Y Liu, N Xiong, Y Zhao, A Vasilakos, J Gao, Y Jia, Multi-layer clustering routing algorithm for wireless vehicular sensor networks. IET Commun. 4(7), 810-816 (2010)

27. Y Zeng, K Xiang, D Li, A Vasilakos, Directional routing and scheduling for green vehicular delay tolerant networks. Wireless Netw. 19(2), 161-173 (2013)

28. T Spyropoulos, R Rais, T Turletti, K Obraczka, A Vasilakos, Routing for disruption tolerant networks: taxonomy and design. Wireless Netw. 16(8), 2349-2370 (2010)

29. M Youssef, M Ibrahim, M Abdelatif, L Chen, A Vasilakos, Routing metrics of cognitive radio networks: a survey. IEEE Commun. Surv. Tutor. 16(1), 92-109 (2014)

30. L Zhou, Y Zhang, K Song, W Jing, A Vasilakos, Distributed media services in p2p-based vehicular networks. IEEE Trans. Vehic. Technol. 60(2), 692-703 (2011)

31. Z Sheng, S Yang, Y Yu, A Vasilakos, McJ Cann, K Leung, A survey on the IETF protocol suite for the internet of things: standards, challenges, and opportunities. IEEE Wireless Commun. 20(6), 91-98 (2013) 
32. L Xiang, J Luo, A Vasilakos, in Proc. IEEE Sensor, Mesh and Ad Hoc Communications and Networks. Compressed data aggregation for energy efficient wireless sensor networks, (2011), pp. 46-54

33. H Cheng, N Xiong, AV Vasilakos, L Tianruo Yang, G Chen, X Zhuang, Nodes organization for channel assignment with topology preservation in multi-radio wireless mesh networks. Ad Hoc Netw. 10(5), 760-773 (2012)

34. P Li, S Guo, S Yu, AV Vasilakos, in Proc. IEEE INFOCOM. Codepipe: an opportunistic feeding and routing protocol for reliable multicast with pipelined network coding, (2012), pp. 100-108

35. JJ Blum, A Eskandarian, A reliable link-layer protocol for robust and scalable intervehicle communications. IEEE Trans. Intell. Transportation Syst. 8(1), 4-13 (2007)

36. M Sepulcre, J Gozalvez, J Harri, H Hartenstein, Contextual communications congestion control for cooperative vehicular networks. IEEE Trans. Wireless Commun. 10(2), 385-389 (2011)

37. Y Park, H Kim, Application-level frequency control of periodic safety messages in the IEEE WAVE. IEEE Trans. Vehic. Technol. 61(4), 1854-1862 (2012)

38. T Kuhn, Jl de Irigon, in Proc. ACM International Workshop on Mobility Management and Wireless Access. An experimental evaluation of black burst transmissions (Crete Island, Greece, 2007), pp. 163-167

39. K Abboud, W Zhuang, in Proc. IEEE Global Telecommunications Conf. Modeling and analysis for emergency messaging delay in vehicular ad hoc networks, (2009), pp. 1-6

40. J Karedal, F Tufvesson, N Czink, A Paier, C Dumard, T Zemen, CF Mecklenbrauker, AF Molisch, A geometry-based stochastic MIMO model for vehicle-to-vehicle communications. IEEE Trans. Wireless Commun. 8(7), 3646-3657 (2009)

41. AF Molisch, F Tufvesson, J Karedal, CF Mecklenbrauker, A survey on vehicle-to-vehicle propagation channels. IEEE Wireless Commun. 16(6), 12-22 (2009)

42. LCheng, BE Henty, DD Stancil, F Bai, P Mudalige, Mobile vehicle-to-vehicle narrow-band channel measurement and characterization of the $5.9 \mathrm{ghz}$ dedicated short range communication (DSRC) frequency band. IEEE J. Select. Areas Commun. 25(8), 1501-1516 (2007)

43. X Cheng, C-X Wang, DI Laurenson, S Salous, A Vasilakos, An adaptive geometry-based stochastic model for non-isotropic MIMO mobile-to-mobile channels. IEEE Trans. Wireless Commun. 8(9), 4824-4835 (2009)

44. National Highway Traffic Safety Administration, Vehicle safety communications project. Task 3 final report: identify intelligent vehicle safety applications enabled by DSRC. Technical report (Washington, DC, 2005)

doi:10.1186/1687-1499-2014-179

Cite this article as: Awais Javed et al:: A multi-hop broadcast protocol design for emergency warning notification in highway VANETs. EURASIP Journal on Wireless Communications and Networking 2014 2014:179.

\section{Submit your manuscript to a SpringerOpen ${ }^{\circ}$ journal and benefit from:}

- Convenient online submission

- Rigorous peer review

- Immediate publication on acceptance

- Open access: articles freely available online

- High visibility within the field

- Retaining the copyright to your article

Submit your next manuscript at $\boldsymbol{\wedge}$ springeropen.com 